\title{
Violencia escolar en adolescentes: un análisis en función de la actividad física y lugar de residencia habitual*
}

\author{
School violence in adolescents: A analysis based \\ on physical activity and place of residence
}

Enviado: octubre 3 de 2013 | Revisado: febrero 5 de 2015 | Aceptado: febrero 5 de 2015

\author{
Felix ZuRITA ORTEGA** \\ Universidad de Granada, España \\ JOSE MARIA VILCHES AZNAR *** \\ Universidad de Granada, España \\ JAVIER CACHON ZAGALAZ ***** \\ Universidad de Jaen, España \\ Rosario PAdial Ruz****** \\ Universidad de Granada, España \\ ASUNCION MARTÍNEZ MARTINEZ ******* \\ Universidad de Granada, España \\ Manuel CASTRO SANCHEZ ******** \\ Universidad de Granada, España
}

doi:10.11144/Javeriana.upsy14-2.veaa

Para citar este artículo: Zurita, F., Vilches, J. M., Cachon, J., Padial, R., Martínez, A., \& Castro, M. (2015). Violencia escolar en adolescentes: un análisis en función de la Actividad Física y Lugar de Residencia Habitual. Universitas Psychologica, 14(2), 743-754. http://dx.doi.org.10.11144/Javeriana. upsy14-2.veaa

\footnotetext{
* Artículo de Investigación

***felixzo@ugr.es

****obelux@hotmail.com

***** jcachon@ujaen.es

******rpadial@ugr.es

******** asuncionmm@ugr.es

********* manue87@correo.ugr.es
}

\section{RES UMEN}

Son variados y frecuentes los estudios que intentan establecer la relación directa entre variables de tipo psicosocial y deportivo en la conducta violenta y agresiva de los adolescentes, pero pocos incluyen la relación con residir fuera del contexto familiar. De esta manera en este estudio se evalúa la agresividad y componentes de la misma en jóvenes adolescentes, determinando los niveles de práctica de actividad físico-deportiva así como el lugar de residencia de alumnos de ESO de la ciudad de Granada (España) y dictaminando posibles relaciones entre la agresividad, lugar de residencia y realización de actividad física continua. La participación de un total de 2.273 adolescentes permitió el registro y evaluación de las variables de conducta violenta (medida con la escala de conducta violenta en la escuela), lugar de residencia y práctica de actividad física. Los resultados mostraron que los adolescentes no residentes en el hogar familiar tenían mayores índices de agresividad que los residentes y que los que practicaban deporte de manera regular presentaban índices de agresividad manifiesta mayores que los sedentarios. Como principal conclusión indicamos la necesidad de elaborar más estudios que intenten dilucidar más detalladamente aquellas variables que pueden inducir a comportamientos agresivos.

Palabras clave

agresividad; adolescente; actividad física; internado; menores

\begin{abstract}
A B S T R A C T
The studies attempting to establish the relationship directly between psychosocial variables and sporty type in violent behavior and aggressive adolescents are varied and frequent, but few include the relationship with reside outside the family context. The aim in this study is evaluated aggressiveness and components thereof in young adolescents, determining practice levels
\end{abstract}


of physical and sporting activities as well as the residence of ESO students from the city of Granada (Spain) ruling and possible relationships between aggressiveness, place of residence and conducting continuous physical activity. The participation of a total 2,273 adolescents allowed the recording and evaluation of variables violent behavior (as measured by the scale of violent behavior at school) place of residence and physical activity. The results showed non-residents adolescents in the family home had higher aggressiveness indexes residents and practicing the sport regularly they had higher rates of aggression manifests that sedentary. The main conclusion indicated the need for develop more studies that attempt to elucidate in more detail those variables that can lead to aggressive behavior.

Keywords

aggressiveness; teenager; physical activity; boarding; children

\section{Introducción}

No cabe duda que dentro de la sociedad actual se ha producido un aumento considerable de denuncias por conductas violentas en la escuela, ante esta situación algunos autores como Anderson \& Bushman (2002) o Garfield \& Llanten (2004), indican que las cifras constituyen una epidemia del siglo XXI; en diversos contextos ha aumentado la sensibilidad hacía esta situación e intentan de una manera fehaciente alcanzar medidas que palíen este hecho.

Entre las posturas adoptadas se encuentran el estudio de las estrategias de afrontamiento y emociones como señalan Mestre, Samper, Tur-Porcar, Richaud de Minzi y Mesurado (2012), otros autores analizan el tipo de agresividad que predomina, por sexo y edad y buscan medidas que aminoren o desfavorezcan esas situaciones (Chahín-Pinzón, Lorenzo-Seva, \& Vigil-Colet, 2011), a estos debemos de unir aquellos que tienen en cuenta aspectos intrínsecos en la escuela como es por ejemplo, el clima escolar, que condiciona de manera directa o indirecta a nuestros escolares (Aron, Milicic, \& Armijo, 2012). Del mismo modo debemos indicar multitud de programas realizados encaminados a la prevención de esta problemática desde hace décadas (Viera, Fernández-García, \& Quevedo, 1989; Cerezo \& Esteban, 1992; Mora-Merchán \& Ortega, 1997; Pareja, 2002).
Asimismo debemos puntualizar cómo la agresividad relacional o indirecta viene caracterizada por una exclusión social basada en rumores, $\mathrm{cu}$ chicheos... como señalan en sus estudios Card, Stucky, Sawalani y Little (2008) y Cava, Buelga, Musitu y Murgui (2010), si bien otros autores no hablan exclusivamente de agresividad relacional sino que la equiparan a la indirecta como toldos (2005), también existe la de tipo manifiesta que presenta unas características de tipo más físico, caracterizado por conductas y comportamientos con la finalidad de dañar al oponente (Ramírez \& Andreu, 2003). Dada la ambigüedad de clasificaciones y denominaciones Estévez (2005), realiza una extensa síntesis de los diversos conceptos en su trabajo "Violencia, victimización y rechazo escolar en la adolescencia".

Uno de los contextos donde se hacen más patentes las diferencias entre jóvenes y adolescentes es el de las residencias escolares o internados que son centros que acogen en régimen de familias sustitutorias, a aquellos jóvenes que cursan sus estudios fuera de su lugar de origen y cuyas situaciones personales o familiares así lo aconsejan, siguiendo el modelo de beneficencia institucional que es común para todas las edades y personas con carencias familiares y socioeconómicas, donde se recogen en grandes centros y macro instituciones a estos menores a cargo de adultos, como señalan Del Valle y Fuertes (2000). Estos centros facilitan el acceso al sistema educativo (estudios obligatorios y posobligatorios) ya sea por cuestiones socioeconómicas, geográficas (población rural diseminados), o por cualquier otra circunstancia familiar y de esta manera favorecen la igualdad de oportunidades y la equidad en la educación, al posibilitar la atención de aquel alumnado que tiene dificultades para acceder a los distintos niveles educativos, por carecer de esa oferta en su lugar de origen.

En España según datos II PENIA (II Plan Estratégico Nacional de Infancia y Adolescencia 2013-2016), se ha producido un incremento del acogimiento residencial propiciado entre otras causas por la crisis económica que arrastramos desde hace años y que impiden en cierto modo que existan familias que no pueden hacerse cargo de los estudios de sus hijos. A eso debemos añadir 
los menores que emigran y a aquellos que tienen a sus progenitores en centros penitenciarios. Estos alumnos con ciertas necesidades educativas, hacen necesario que se investigue y fomente la mejor manera de hacer llevadera su estancia. Así estudios como los de Fernández-Molina, Del Valle, Fuentes, Bernedo y Bravo (2011) tratan de conocer si existen diferencias en problemas de conducta, según el tipo de acogimiento en que se encuentra el adolescente (preadoptivo, residencial o con abuelos) y estudios como los de Del Valle y Bravo (2009) inciden sobre la intervención socioeducativa en dicho acogimiento residencial.

En el anteriormente mencionado Plan Estratégico Nacional (2013) indican la importancia de la formación en valores y es desde los programas de educación física que se encuentra el lugar ideal para su fomento (Collado, 2005), puesto que constituye un importante factor de mejora del rendimiento escolar. Durante el desarrollo de estos programas es donde los adolescentes crean las bases para un nuevo modelo de toma de decisiones. Por lo tanto debemos favorecer entornos seguros para los adolescentes donde se incluyan estrategias que aseguren un gasto energético y un control de los impulsos. Según Vidal y Mota (2008) el deporte es uno de los medios y recursos que salva al mundo asociativo y socializante, tanto en la infancia como en la adolescencia y en la vida adulta y señalan que la actividad física entre los adolescentes es la actividad más demandada.

En estudios como los de Pelegrín (2004) y Chahín-Pinzon y Libia (2011) se analiza la relación entre agresividad y actividad física en adolescentes, observándose los beneficios, tanto fisiológicos como psicológicos que nos aporta la actividad física y cómo esta favorece el control de impulsos y por tanto los niveles de agresividad.

En nuestro caso uno de los elementos que considerábamos importantes dada la escasez de estudios sobre la temática, era constatar si los alumnos que se encuentran en régimen de internado (no vivían con sus padres) poseían niveles de agresividad mayores hacía sus compañeros que sí residían en el domicilio familiar. En relación a este último aspecto, el presente estudio nos aporta datos sobre lo que acontece en adolescentes de 13 a 17 años en relación a conductas violentas y si estas predominan más en alumnos/as internos o practicantes asiduos de actividad física. Nos hemos planteado como objetivos:

- Evaluar la agresividad y componentes de la misma en jóvenes adolescentes.

- Determinar los niveles de práctica de actividad físico-deportiva así como el lugar de residencia de alumnos de ESO de la ciudad de Granada (España).

- Dictaminar si la agresividad o conducta violenta vienen establecidas por el lugar de residencia y por la realización de actividad física continua.

\section{Material y Métodos}

\section{Participantes}

La selección de los participantes se genera del total de 11.030 alumnos/as matriculados en la Educación Secundaria Obligatoria (datos facilitados por la Consejería de Educación de la Junta de Andalucía) durante el curso académico 2012/2013 en la capital de Granada. Para establecer una muestra representativa (error al 0.02; I.C $=95.5 \%$ ), se realizó mediante técnicas de estratificación, proporcionalidad y aleatorización, considerándose tipo de centro (concertado o público), sexo (hombres-mujeres) y ciclo (primero-segundo). Participaron en esta investigación un total de 2.273 adolescentes de Granada (España), con una edad comprendida entre los 13 y 17 años $(M=14.94$ años; DT= 1.258). El estudio cumplió con las normas éticas del Comité de Investigación y Declaración de Helsinki de 1975. Se contó en todos los casos con el consentimiento informado de los participantes y se respetó el resguardo a la confidencialidad.

\section{Diseño}

En este estudio se utilizó un diseño de carácter cuantitativo descriptivo y de tipo transversal. Posteriormente se desarrolló un estudio relacional para 
analizar el grado de dependencia entre las distintas variables objeto de estudio.

\section{Variables e instrumentos}

La presente investigación tomó como referencia las siguientes variables e instrumentos de medida, es decir:

- Género: según fuese masculino o femenino.

- Conducta violenta en la escuela: dividida en dos categorías: agresión manifiesta o directa; o agresión relacional o indirecta, subdividida a su vez cada una de ellas en tres sub-escalas (pura, reactiva o instrumental) analizado mediante la escala de conducta violenta en la escuela propuesta en su versión original por Little, Henrich, Jones y Hawley (2003), adaptada por el Grupo Lisis (Estévez, 2005) y empleada en estudios de similares características entres otros por Cava, Musitu y Murguy (2006), Musitu, Estévez y Emler (2007) o Jiménez, Moreno, Murguy y Musitu (2008). Se compone de una escala tipo Likert de 25 ítems que oscila entre los valores 1(nunca) al 4 (siempre), que una vez puntuados nos dan dos tipos de conducta violenta: agresión manifiesta o directa (la cual se genera en un encuentro cara a cara donde el agresor es identificable por la victima) o agresión relacional o indirecta (considerada cuando el agresor permanece de una forma anónima). Presentan un coeficiente de fiabilidad Alphade Cronbach de 0.824 para los ítems que miden la agresividad manifiesta y 0.722 para las cuestiones de la agresividad relacional, muy similares al 0.088 y 0.081 , para ambas sub escalas obtenido por Musitu et al., (2007).

- Actividad física: con la opción de si práctica o no, utilizándose una hoja de registro para ello, donde se instaba a marcar la opción de Sí, siempre y cuando el adolescente realizase actividad física fuera del centro escolar con una frecuencia igual o superior a tres horas semanales.
- Lugar de residencia mientras estudia: categorizada en domicilio familiar o residencia escolar y registrado mediante hoja de registro.

\section{Procedimiento}

En primer lugar, a través de la Facultad de Ciencias de la Educación de la Universidad de Granada (área de corporal), y en contacto con la delegación de educación de la Junta de Andalucía, se solicitó la colaboración de los centros educativos de la capital de Granada seleccionados a partir de un muestreo de conveniencia de las categorías objeto de estudio. La dirección de cada centro educativo fue informada sobre la naturaleza de la investigación y se le solicitó la colaboración de sus alumnos/as. En segundo lugar se adjuntó un modelo de autorización destinado a los responsables legales de los adolescentes pidiéndoles su consentimiento informado.

En todo momento se garantizó a los participantes el anonimato de la información recogida aclarando que su utilización sería solo con fines científicos. Los encuestadores estuvieron presentes durante la recogida de los datos para poder resolver cualquier duda al respecto. La recogida se desarrolló sin ningún tipo de problema o anormalidad a reseñar. En último lugar se agradeció a los docentes, orientadores y responsables su colaboración y se les informó del envío en un futuro próximo de un informe sobre los datos obtenidos respetando la confidencialidad de los mismos.

\section{Análisis de los datos}

Para el análisis de los datos se utilizó el programa estadístico SPSS 20.0., mediante la utilización de descriptivos, medias y ANOVA para establecer las diferencias entre agresividad, actividad física y lugar de residencia habitual.

\section{Resultados}

Tomando como referencia los análisis descriptivos, concernientes a los 2.273 adolescentes, respecto a las variables seleccionadas, se constató 
que $1.208(53.1 \%)$ adolescentes eran varones y 1.065 (46.9\%) eran mujeres. La mayor parte de ellos residían en el domicilio familiar (91.1\%; $\mathrm{n}=2070$ ), únicamente 203 adolescentes tenían su domicilio en residencia o internado (8.9\%), asimismo $1.823(80.2 \%)$ practicaban actividad física de forma habitual al menos tres horas semanales fuera del contexto escolar frente al restante $19.8 \%(n=450)$ que no la hacían. Los descriptivos concernientes a la conducta violenta obtuvieron los siguientes resultados (Tabla 1), todos ellos presentaron valores entre 1 (nunca) y 2 (pocas veces).

En cuanto a los valores según cada una de las sub escalas concernientes a la agresividad (Tabla 2) se registraron valores similares entre agresividad mani- fiesta y relacional ( $\mathrm{M}=1.29$ y 1.26 respectivamente) y dentro de cada una de ellas la distribución de las sub escalas es similar pues la que mayor valor medio presenta es la de tipo reactivo, seguido de la pura y por último la instrumental como puede apreciarse en la siguiente tabla:

En lo concerniente al estudio relacional que nos permitirá establecer el tipo de conexión entre la conducta violenta y la actividad física y lugar donde residen habitualmente, los datos mostraron en primer lugar que en referencia a la actividad física y el lugar de residencia (Tabla 3), no se hallaron diferencias estadísticamente significativas $(p=0.556)$.

En cuanto a la conducta violenta y lugar de residencia habitual, debemos señalar que tanto en la

Tabla 1. Descriptivos de los ítems de la escala de conducta violenta

\begin{tabular}{|c|c|c|}
\hline Test de conducta violenta & Media & Desv. típ. \\
\hline I.1. Soy una persona que se pelea con los demás & 1.34 & 0.555 \\
\hline I.2. Cuando alguien me hace daño o me hiere, le pego & 1.62 & 0.824 \\
\hline I.3. Amenazo a otros/as para conseguir lo que quiero & 1.10 & 0.371 \\
\hline I.4. Soy una persona que dice a sus amigos/as que no se relacionen o salgan con otros/as & 1.13 & 0.380 \\
\hline I.5. Si alguien me enfada o me hace daño, digo a mis amigos que no se relacionen con esa persona & 1.26 & 0.536 \\
\hline I. 6. Para conseguir lo que quiero digo a mis amigos/as que no se relacionen o salgan con otros/as & 1.09 & 0.321 \\
\hline I.7. Soy una persona que pega, da patadas y puñetazos a los demás & 1.19 & 0.495 \\
\hline I.8. Cuando alguien me amenaza, yo le amenazo también & 1.67 & 0.890 \\
\hline I.9. Pego, doy patadas o puñetazos para conseguir lo que quiero & 1.11 & 0.404 \\
\hline I.10. Soy una persona que no deja a los demás que entren en su grupo de amigos/as. & 1.11 & 0.380 \\
\hline I.11.Si alguien me hace daño o me hiere, no dejo que esa persona forme parte de mi grupo de amigos/as & 1.92 & 1.023 \\
\hline I.12. Para conseguir lo que quiero, no dejo que algunas personas formen parte de mi grupo de amigos/as & 1.15 & 0.423 \\
\hline I.13. Soy una persona que dice cosas malas y negativas a los demás & 1.25 & 0.496 \\
\hline I.14. Cuando alguien me enfada, le pego, le pataleo o le doy puñetazos & 1.26 & 0.581 \\
\hline I.15. Para conseguir lo que quiero, digo cosas malas y negativas a los demás (insulto & 1.09 & 0.335 \\
\hline I.16. Soy una persona que trata con indiferencia a los demás o deja de hablar con ellos & 1.24 & 0.524 \\
\hline I. 17. Soy una persona que chismorrea y cuenta rumores de los demás & 1.70 & 0.838 \\
\hline I.18. Para conseguir lo que quiero, trato con indiferencia o dejo de hablar con algunas personas & 1.18 & 0.492 \\
\hline I.19. Soy una persona que desprecia a los demás & 1.13 & 0.416 \\
\hline I.20. Cuando alguien consigue enfadarme, le hago daño o le hiero & 1.42 & 0.720 \\
\hline I.21. Para conseguir lo que quiero, desprecio a los demás & 1.24 & 0.483 \\
\hline I.22. Soy una persona que chismorrea o cuenta cosas sobre los demás & 1.30 & 0.570 \\
\hline I.23. Cuando alguien me enfada, chismorreo o cuento rumores sobre esa persona & 1.29 & 0.551 \\
\hline I.24. Para conseguir lo que quiero, chismorreo o cuento rumores sobre los demás- & 1.11 & 0.357 \\
\hline I. 25. Para conseguir lo que quiero, hago daño o hiero a los demás & 1.14 & 0.416 \\
\hline
\end{tabular}

Fuente: elaboración propia 
Felix Zurita Ortega, Jose Maria Vilches Aznar, Javier Cachon Zagalaz, Rosario Padial Rus, Asuncion Martínez Martinez, Manuel Castro Sanchez

TABLA 2. Distribución de las sub escalas de la conducta violenta

\begin{tabular}{cccccc}
\hline Agresividad & N & Mínimo & Máximo & Media & Desv. Típ. \\
\hline Agresividad manifiesta o directa & 2273 & 1.000 & 4.000 & 1.29 & 0.55 \\
Agresividad manifiesta pura & 2273 & 1.000 & 4.000 & 1.22 & 0.49 \\
Agresividad manifiesta reactiva & 2273 & 1.000 & 4.000 & 1.56 & 0.80 \\
Agresividad manifiesta instrumental & 2273 & 1.000 & 4.000 & 1.13 & 0.40 \\
Agresividad relacional o indirecta & 2273 & 1.000 & 4.000 & 1.26 & 0.51 \\
Agresividad relacional pura & 2273 & 1.000 & 4.000 & 1.19 & 0.46 \\
Agresividad relacional reactiva & 2273 & 1.000 & 4.000 & 1.46 & 0.68 \\
Agresividad relacional instrumental & 2273 & 1.000 & 4.000 & 1.13 & 0.39 \\
\hline
\end{tabular}

Fuente: elaboración propia

Tabla 3. Lugar de residencia y práctica de actividad física

\begin{tabular}{|c|c|c|c|c|}
\hline \multirow{2}{*}{\multicolumn{2}{|c|}{ Resides }} & \multicolumn{2}{|c|}{$\mathrm{AF}$} & \multirow{3}{*}{$\begin{array}{l}\text { Total } \\
2070\end{array}$} \\
\hline & & \multirow{2}{*}{$\begin{array}{c}\mathrm{Si} \\
1657\end{array}$} & \multirow{2}{*}{$\begin{array}{l}\text { No } \\
413\end{array}$} & \\
\hline Domicilio familiar & Recuento & & & \\
\hline & \% de Resides & $80.0 \%$ & $20.0 \%$ & $100 \%$ \\
\hline & $\%$ de AF & $90.9 \%$ & $91.8 \%$ & $91.1 \%$ \\
\hline \multirow[t]{3}{*}{ Residencia } & Recuento & 166 & 37 & 203 \\
\hline & $\%$ de Resides & $81.8 \%$ & $18.2 \%$ & $100 \%$ \\
\hline & $\%$ de $\mathrm{AF}$ & $9.1 \%$ & $8.2 \%$ & $8.9 \%$ \\
\hline \multirow[t]{3}{*}{ Total } & Recuento & 1823 & 450 & 2273 \\
\hline & \% de Resides & $80.2 \%$ & $19.8 \%$ & $100 \%$ \\
\hline & $\%$ de $\mathrm{AF}$ & $100 \%$ & $100 \%$ & $100 \%$ \\
\hline
\end{tabular}

Fuente: elaboración propia

de tipo manifiesta como relacional se detectó falta de correspondencia ( $\left.\mathrm{p} \leq 0.05^{* *}\right)$, propiciado porque en la inmensa mayoría de las cuestiones planteadas los participantes que vivían en centros con internados presentaron valores medios más altos en todas las sub escalas, como se puede apreciar en la siguiente tabla:

Tomando ahora como referencia los análisis de la conducta violenta en base a la realización de actividad física, se hallaron diferencias estadísticamente significativas $\left(p \leq 0.005^{* *}\right)$ en agresividad manifiesta y sus tres sub escalas, derivadas de los adolescentes practicantes asiduos de actividad física que presentaron valores más altos que los no practicante. En lo que respecta a la agresividad relacional, no deparó diferencias entre los que hacían o no actividad física, como se puede observar en la siguiente tabla (Tabla 5):

\section{Discusión}

En esta investigación se han analizado las repercusiones que tiene la práctica de actividad física de forma continua y el lugar de vivienda habitual en adolescentes, partiendo de la importancia que actualmente la sociedad, medios de comunicación y estamentos familiares-educativos le conceden al comportamiento agresivo de los adolescentes. No cabe duda que es necesario conocer aquellos elementos que pudiesen condicionar esta situación. Nuestro estudio se realizó con 2,273 jóvenes, es similar a otros realizados en la geografía española (Gázquez, Pérez-Fuentes, Carrión, \& Santiuste, 2009; Mestre et al., 2012; Torregrosa, Inglés, García-Fernández, Valle, \& Núñez, 2012), trabajos encaminados en su totalidad a dar respuesta a proyectos educativos sobre conducta violenta en los centros escolares. 
VIOLENCIA ESCOLAR EN ADOLESCENTES: UN ANÁLISIS EN FUNCIÓN DE LA ACTIVIDAD FÍSICA Y LUGAR DE RESIDENCIA HABITUAL

Tabla 4. Distribución de los ítems de conducta violenta en función de lugar de residencia habitual

\begin{tabular}{|c|c|c|c|c|c|c|}
\hline Lugar de Residencia & & $\mathrm{N}$ & Media & D.T & Error típo de la media & Sig. \\
\hline \multirow{2}{*}{ Agresividad manifiesta } & Domicilio familiar & 2070 & 1.2886 & 0.32403 & 0.00712 & \multirow{2}{*}{$0.001 * *$} \\
\hline & Residencia & 203 & 1.3831 & 0.40131 & 0.02817 & \\
\hline \multirow{2}{*}{ Agresividad manifiesta pura } & Domicilio familiar & 2070 & 1.2175 & 0.33711 & 0.00741 & \multirow{2}{*}{$0.000 * *$} \\
\hline & Residencia & 203 & 1.3374 & 0.46411 & 0.03257 & \\
\hline \multirow{2}{*}{ Agresividad manifiesta reactiva } & Domicilio familiar & 2070 & 1.5580 & 0.56604 & 0.01244 & \multirow{2}{*}{$0.026 * *$} \\
\hline & Residencia & 203 & 1.6515 & 0.62400 & 0.04380 & \\
\hline \multirow{2}{*}{ Agresividad manifiesta instrumental } & Domicilio familiar & 2070 & 1.1299 & 0.27379 & 0.00602 & \multirow{2}{*}{$0.000 * *$} \\
\hline & Residencia & 203 & 1.2049 & 0.33386 & 0.02343 & \\
\hline \multirow{2}{*}{ Agresividad relacional } & Domicilio familiar & 2070 & 1.2584 & 0.26216 & 0.00576 & \multirow{2}{*}{$0.002 * *$} \\
\hline & Residencia & 203 & 1.3321 & 0.32077 & 0.02251 & \\
\hline \multirow{2}{*}{ Agresividad relacional pura } & Domicilio familiar & 2070 & 1.1905 & 0.27990 & 0.00615 & \multirow{2}{*}{0.066} \\
\hline & Residencia & 203 & 1.2352 & 0.33342 & 0.02340 & \\
\hline \multirow{2}{*}{ Agresividad relacional reactiva } & Domicilio familiar & 2070 & 1.4618 & 0.42451 & 0.00933 & \multirow{2}{*}{$0.030 * *$} \\
\hline & Residencia & 203 & 1.5406 & 0.49741 & 0.03491 & \\
\hline \multirow{2}{*}{ Agresividad relacional instrumental } & Domicilio familiar & 2070 & 1.1229 & 0.26240 & 0.00577 & \multirow{2}{*}{$0.000 * *$} \\
\hline & Residencia & 203 & 1.2204 & 0.33150 & 0.02327 & \\
\hline
\end{tabular}

Fuente: elaboración propia

Tabla 5. Distribución de los ítems de conducta violenta en función de la práctica de actividad física

\begin{tabular}{llccccc}
\hline \multicolumn{2}{c}{ Práctica de actividad física } & $\mathrm{N}$ & Media & D.T & Error típo de la media & Sig. \\
\hline \multirow{2}{*}{ Agresividad manifiesta } & Sí Practica & 1823 & 1.2357 & 0.35990 & 0.00843 & $0.001^{* *}$ \\
& No Practica & 450 & 1.1978 & 0.31578 & 0.01489 & \\
Agresividad manifiesta pura & Sí Practica & 1823 & 1.5802 & 0.57824 & 0.01354 & $0.040^{* *}$ \\
& No Practica & 450 & 1.5100 & 0.54263 & 0.02558 & \\
Agresividad manifiesta reactiva & Sí Practica & 1823 & 1.1426 & 0.28849 & 0.00676 & $0.015^{* *}$ \\
& No Practica & 450 & 1.1120 & 0.24374 & 0.01149 & \\
Agresividad manifiesta instrumental & Sí Practica & 1823 & 1.3059 & 0.34135 & 0.00799 & \multirow{2}{*}{$0.022^{* *}$} \\
& No Practica & 450 & 1.2609 & 0.29239 & 0.01378 & \\
Agresividad relacional & Sí Practica & 1823 & 1.2637 & 0.26822 & 0.00628 & \multirow{2}{*}{0.649} \\
& No Practica & 450 & 1.2702 & 0.27066 & 0.01276 & \\
Agresividad relacional pura & Sí Practica & 1823 & 1.1925 & 0.28840 & 0.00675 & 0.519 \\
& No Practica & 450 & 1.2022 & 0.27253 & 0.01285 & \\
Agresividad relacional reactiva & Sí Practica & 1823 & 1.4708 & 0.43562 & 0.01020 & 0.670 \\
& No Practica & 450 & 1.4611 & 0.41732 & 0.01967 & \\
Agresividad relacional instrumental & Sí Practica & 1823 & 1.1278 & 0.26346 & 0.00617 & 0.206 \\
& No Practica & 450 & 1.1472 & 0.29776 & 0.01404 & \\
\hline
\end{tabular}

Fuente: elaboración propia

En España se vienen produciendo cambios desde hace más de veinticinco años destinados a modernizar la red de protección a la infancia. (Del Valle, López, Montserrat, \& Bravo, 2009; De Paul, 2009; Del Valle, Bravo, \& López, 2009); en la ciudad de
Granada, uno de cada diez alumnos/as en edad adolescente se encuentra en régimen de internado. Este dato confirma lo reseñado por el Plan Estratégico de Infancia y Adolescencia 2013-2016 que indicaba que una población total de 14.088 niños se 
encuentran en acogimiento residencial en España, indicando que factores de índole económico, familiar o académicos incidían en estas cifras y que cada vez van en aumento (Del Valle \& Fuertes, 2000).

Prestando ahora atención a la variable actividad física, es preciso indicar que uno de cada cinco participantes señalaban la no realización de forma habitual al menos durante tres horas semanales fuera del contexto escolar de dicha actividad, esto confirma lo mostrado por Chahín-Pinzón y Libia (2011) que señalaban como motivo de abandono las nuevas tecnologías. También Casado, Alonso, Hernández, Barrera y Jiménez (2009) establecían que a estas edades las adolescentes femeninas disminuían la práctica regular de actividad física.

Tomando en consideración los resultados que muestran las conductas violentas de los adolescentes, debemos indicar que tanto la de tipo manifiesta como relacional se postulan con valores muy similares; ambas funcionan como respuesta defensiva ante alguna provocación y dentro de las sub escalas, son las reactivas las más generalizadas al igual que señalan Ramos-Corpas y Musitu, (2007). Rose, Swenson y Waller (2004) aluden a que en estas edades aparece un aumento de percepción de la popularidad por el uso de la violencia relacional, sobre todo entre las chicas, y cuya finalidad no es otra que la de mejorar su estatus social y en cambio, la violencia manifiesta se da más entre los varones ya que conlleva contacto físico entre las partes, víctima y agresor, cursándose la reacción ante cualquier tipo de agresividad (Moreno, Estévez, Murgui, \& Musitu, 2009).

Los factores biológicos, psicológicos y sociales forman una red de interacciones contenidas en la actividad humana por tanto como manifiestan Araujo y Díaz (2000), la agresividad se encuentra fundamentada en parámetros y condiciones individuales, grupales, sociales, históricas y culturales. Al hilo de este comentario y coincidiendo con nuestros datos, la agresividad en todas sus variantes se presenta en cifras similares. Valadez y Martín del Campo (2008) señalan la importancia que tiene para erradicar la conducta violenta en el trabajo participativo como respuesta para disminuir el maltrato entre iguales y promover la convivencia educativa, ya sea en el contexto escolar o de residencia estudiantil (Ortega \& Mora-Merchán, 2000).

En lo concerniente al estudio relacional que nos permitirá establecer el tipo de conexión entre la conducta violenta y la actividad física y lugar donde residen habitualmente, los datos mostraron que en referencia a la actividad física y el lugar de residencia, no se encontró asociación, lo que denota que la práctica de actividad física está generalizada en la población adolescente, ya que en los últimos años se ha prestado más atención e importancia a las intervenciones comunitarias para la promoción de la actividad física por el bienestar físico y mental que generan en las personas, como indican Bauman y Graig (2005).

Nuestros resultados sugieren que la conducta violenta en todas las sub escalas es mayor en adolescentes que no residen en el domicilio familiar y son coherentes con los constatados en investigaciones previas que indican que este tipo de participantes podrían mostrar inclinación hacia conductas y comportamientos violentos (Rodríguez, 2004) puesto que el adolescente en relación con sus iguales pretende conseguir una reputación social, reconocimiento o status; al hilo de lo indicado por Povedano, Estévez, Martínez y Monreal (2012) se hace patente lo importante de analizar poblaciones adolescentes fuera del contexto escolar.

Que existan más conductas violentas en centros residenciales de adolescentes es causa de factores múltiples. Debemos considerar que estos jóvenes se encuentran en el centro por falta de recursos económicos de sus familias, por problemas de reclusión de sus progenitores, por problemas de emigración, por maltrato familiar, por abandono y otros causas que estigmatizan y marcan psicológicamente de manera negativa y a veces muy profunda a esas edades y que si a todo eso unimos que los adolescentes empiezan a buscar un estatus entre sus iguales y que muchos de los agresores han sido víctimas anteriormente; tenemos unos adolescentes a la defensiva, pero la residencia escolar debe ser vista no exclusivamente como un lugar para residir sino que debe de incluir el concepto de comunidad de convivencia, fomentando la interacción entre conductas y actitudes (Valadez \& Martín del Campo, 2008). 
Aquellos adolescentes que practicaban actividad física de una forma continua mostraron niveles de agresividad manifiesta más altos que los que no la realizaban, esto es debido a que los adolescentes practicantes asiduos del deporte empiezan a competir. Perder para ellos es frustrante, se preocupan de demostrar mejor habilidad que los demás, todo esto hace que se establezca una relación entre resultado y agresión como señalan en sus estudios Duda, Olson y Templin (1991) y Duda y Huston (1995), trasladando esa competitividad a su vida social de una manera inconsciente. Gómez (2007) señala que la violencia y su actitud ante ella en el contexto físico deportivo tiene solución y es importante que todos los estamentos educativos fomenten su erradicación, ya que la práctica deportiva genera un alto potencial de autoestima y desarrollo social en los adolescentes y es un medio para controlar valores que actúan en beneficio de la sociedad, tal como lo muestran Weinberg y Gould (1996), Pelegrín, Martínez y Garcés de los Fayos (2000) y Pelegrín (2004).

En general consideramos que este trabajo complementa y aporta nuevas perspectivas al estudio de las conductas violentas de los adolescentes y añade nuevas variables a considerar. Asimismo entendemos que entre las limitaciones que presenta el estudio se encuentra la de no incluir sub escalas dentro de los parámetros de actividad física (si la actividad es individual o colectiva o tipo de actividad) y pone de manifiesto para próximos proyectos la importancia de incluir algún agente de intervención (deporte) como medio de prevención de la agresividad en los adolescentes.

\section{Conclusiones}

Como principales conclusiones de este estudio, debemos señalar que la agresividad de tipo manifiesta y relacional obtuvo datos similares y que dentro de cada una de ellas la de tipo reactiva es la más propensa hacia la negatividad. Asimismo se constató que uno de cada diez adolescentes no vivía en residencia familiar y mostraron niveles de agresividad muy altos en comparación con los que sí residían en el hogar familiar. Los índices de práctica de actividad física señalaron que cuatro de cada cinco adolescentes regularmente realizaban deporte y estos practicantes mostraron conductas agresivas de tipo manifiesto muy superiores a los adolescentes sedentarios:

\section{Bibliografía}

Anderson, C. A., \& Bushman, B. (2002). Human Aggression. Annual Review of Psychology, 53, 27-51.

Araujo, R., \& Díaz, G. (2000). Un enfoque teórico metodológico para el estudio de la violencia. Revista Cubana de Salud Pública, 24(2), 85-90.

Aron, A. M., Milicic, N., \& Armijo, I. (2012). Clima Social Escolar: una escala de evaluación -Escala de Clima Social Escolar, ECLIS-. Universitas Psychologica, 11(3), 803-813.

Bauman, A., \& Craig, C. L. (2005). The place of physical in the Who Globaln Strategy on Diet and Physical Activity. International Journal of Behavioral Nutrition and Physical Activity, 2,10.

Card, N. A., Stucky, B. D., Sawalani, G. M., \& Little, T. D. (2008). Direct and indirect aggression during childhood and adolescence: A meta-analytic review of gender differences, intercorrelations, and relations to maladjustment. Child Development, 79(5), 1185-1229.

Casado, C., Alonso, N., Hernández, V., Barrera, A., \& Jiménez, R. (2009). Actividad física en niños españoles. Factores asociados y evolución 2003-2006. Revista Pediátrica Atención Primaria, 11 ,219-231.

Cava, M. J., Buelga, S., Musitu, G., \& Murgui, S. (2010). Violencia escolar entre adolescentes y sus implicaciones en el ajuste psicosocial: un estudio longitudinal. Revista de Psicodidáctica, 15(1), 21-34.

Cava, M. J., Musitu, G., \& Murgui, S. (2006). Familia y violencia escolar: el rol mediador de la autoestima y la actitud hacia la autoridad institucional. Psicothema, 18, 367-373.

Cerezo, F., \& Esteban, M. (1992). La dinámica bully-víctima entre escolares. Diversos enfoques metodológicos. Revista de Psicología Universitas Tarraconensis, 4(2), 131-145.

Chahín-Pinzón N., \& Libia, B. (2011). Actividad física en adolescentes y su relación con agresividad, 
Felix Zurita Ortega, Jose Maria Vilches Aznar, Javier Cachon Zagalaz, Rosario

Padial Rus, Asuncion Martínez Martinez, Manuel Castro Sanchez

impulsividad, Internet y videojuegos. Psychologia: Avances de la Disciplina, 5(1), 9-23.

Chahín-Pinzón, N., Lorenzo-Seva U., \& Vigil-Colet, A. (2012). Características psicométricas de la adaptación colombiana del Cuestionario de Agresividad de Buss y Perry en una muestra de preadolescentes y adolescentes de Bucaramanga. Universitas Psychologica, 11(3), 979-988.

Collado, D. (2005). Transmisión y adquisición de valores a través de un programa de Educación Física basado en el juego motor, en un grupo de alumnos y alumnas de Primero de la ESO. Tesis Doctoral no publicada. Universidad de Granada.

De Paúl, J. (2009). La intervención psicosocial en protección infantil en España: evolución y perspectivas. Papeles del Psicólogo 1, 4-12.

Del Valle, J. F., \& Fuertes, J. (2000). El acogimiento residencial en protección a la infancia. Madrid: Pirámide.

Del Valle J., \& Bravo A. (2009). Intervención socioeducativa en Acogimiento residencial. Dirección General de Políticas Sociales y Centro de Estudios de la Administración Pública Regional de Cantabria. Santander: Gobierno de Cantabria. Disponible en: http://www.serviciossocialescantabria.org/uploads/documentos\%20e\%20informes/Interv\%20 Socioeducativa_Acogimiento\%20Residencial.pdf

Del Valle, J. F., Bravo, A., \& López, M. (2009). El acogimiento familiar en España: implantación y retos actuales. Papeles del Psicólogo, 30(1), 33-41.

Del Valle, J. F., López, M., Montserrat, C., \& Bravo, A. (2009). Twenty years of foster care in Spain: Profiles, patterns and outcomes. Children and Youth Services Review, 31, 847-853.

Duda, J. L., Olson, L. K., \& Templin, T. J. (1991). The relation of task and ego orientations to sportsmanship attitudes and the perceived legitimacy of injurius acts. Research Quaterly for Exercise and Sport, 62, 79-87

Duda, J. L., \& Huston, L. (1995). The relationship of goal orientation and degree of competitive sport participation to the endorsement of aggressive acts in American football. IXth Europen Congress on Sport Psychology, 665-662.
Estévez, E. (2005). Violencia, victimización y rechazo escolar en la adolescencia. Universitat de Valencia: Servei de publicacions.

Fernández-Molina M., Del Valle J., Fuentes M. J., Bernedo I., \& Bravo A. (2011). Problemas de conducta de los adolescentes en acogimiento preadoptivo, residencial y con familia extensa. Psicothema, 23(1), 1-6.

Garfield, R., \& Llanten C. (2004). The public health context of violence in Colombia. Revista Panamericana Salud Pública, 16(4), 266-271.

Gázquez, J. J., Pérez-Fuentes, M. C., Carrión, J. J., \& Santiuste, V. (2009). Estudio y análisis de conductas violentas en Educación Secundaria en España. Universitas Psychologica, 9(2), 371-380.

Gómez, A. (2007). La violencia en el deporte: un análisis desde la psicología social. Revista de Psicología Social, 22(1), 63-87.

II Plan Estratégico Nacional de Infancia y Adolescencia 2013-2016.(II PENIA). Gobierno de España. Ministerio de Sanidad, Servicios Sociales e Igualdad. Disponible en: www.observatoriodelainfancia. msssi.gob.es/documentos/PENIA_2013-2016.pdf

Jiménez, T. I., Moreno, D., Murgui, S., \& Musitu, G. (2008). Factores psicosociales relacionados con el estatus social del alumno en el aula: El rol de la reputación social, la amistad, la conducta violenta y la relación con el profesor. International Journal of Psychology and Psychologica Therapy, 8,227-236.

Little, T. D., Henrich, C. C., Jones, S. M. \& Hawley, P. H. (2003). Disentangling the "whys" from the "whats" of aggressive behaviour. International Journal of Behavioral Development, 27,122-133.

Mestre, V., Samper, P., Tur-Porcar, A. M., Richaud de Minzi, M. C., \& Mesurado, B. (2012). Emociones, estilos de afrontamiento y agresividad en la adolescencia. Universitas Psychologica, 11(4), 1263-1275.

Mora-Merchán, J. A., \& Ortega, R. (1997). El Proyecto Sevilla Antiviolencia Escolar: un modelo ecológico de intervención educativa contra el maltrato entre iguales. Conductas agresivas en la edad escolar. Madrid: Pirámide.

Moreno, D., Estévez, E., Murgui, S., \& Musitu, G. (2009). Reputación social y violencia relacional en adolescentes: el rol de la soledad, la autoestima y la satisfacción vital. Psicothema, 21, 537-542. 
Musitu, G., Estévez, E., \& Emler, N. (2007). Adjustment problems in the family and school contexts, attitude towards authority and violent behaviour at school in adolescence. Adolescence, 42, 779-794.

Ortega, R., \& Mora-Merchán, J. A. (2000). Violencia escolar. Mito o realidad. Sevilla: Mergablum.

Pareja, J. A. (2002). La violencia escolar en contextos interculturales. Un estudio de la ciudad autónoma de Ceuta. Tesis Doctoral no publicada: Universidad de Granada.

Pelegrín, A., Martínez, F., \& Garcés de los Fayos, E. J. (2000). La actividad física y el deporte como factores controladores de conductas antisociales y delictivas. II Congreso Iberoamericano de Psicología del Deporte. Huelva: Dirección General de Deportes CARM.

Pelegrín, A. (2004). El comportamiento agresivo y violento: Factores de riesgo y protección como mediadores de inadaptaciones y adaptaciones en la socialización del niño y el adolescente. Tesis Doctoral no publicada: Universidad de Murcia.

Povedano, A., Estévez, E., Martínez, B., \& Monreal, M. C. (2012). Un perfil psicosocial de adolescentes agresores y victimas en la escuela: análisis de las diferencias de género. Revista de Psicología Social, 27(2), 169-182.

Ramírez, J. M., \& Andreu, J. M. (2003). Aggression's typologies. International Review of Social Psychology, 16,125-141

Ramos-Corpas, M. J., \& Musitu, G. (2007). Violencia escolar. Un análisis exploratorio. Investigación DEA. UPO. Sevillla
Rodríguez, N. (2004). Guerra en las aulas. Madrid: Temas de Hoy.

Rose, A. L., Swenson, L.P., \& Waller, E.M. (2004). Overt and relational aggression and perceived popularity: Developmental differences in concurrent and prospective relations. Developmental Psychology, 40, 378-387.

Toldos, M. P. (2005). Sex an age difference in self-estimated physical, verbal and indirect agressive in Spanish adolescent. Aggressive Behavior, 31, 13-23.

Torregrosa, M., S., Inglés C. J., García-Fernández, J. M., Valle, A., \& Núñez J. C. (2012). Relaciones entre conducta agresiva y metas académicas: estudio con una muestra de estudiantes españoles de Educación Secundaria Obligatoria. Universitas Psychologica, 11(4), 1303-1315.

Valadez, I., \& Martín del Campo, S. (2008). El trabajo participativo con docentes: Una búsqueda de propuestas para enfrentar el maltrato entre iguales. Revista Mexicana de Investigación Educativa, 13(136), 87-111.

Vidal, F., \& Mota, R. (2008). Encuesta de Infancia en España 2008. Fundación SM, Universidad Pontificia Comillas-ICAI-ICADE y el Movimiento Junior AC. Madrid

Viera, M., Fernández-García, I., \& Quevedo, G. (1989). Violence, bullyng and counselling in the ibericanpenínsula. En E. Roland, \& E. Munthe (Eds.). Bullyng: A international perspective. London: David Fulton.

Weinberg, R. S., \& Gould, D. (1996). Fundamentos de Psicología del Deporte y el Ejercicio Físico. Barcelona: Ariel Psicología. 
